\title{
Effect of Adipic Acid Concentration on the Isotactic Polypropylene Flat Sheet Using Methyl Salicylate Prepared by TIPS for $\mathrm{CO}_{2} / \mathrm{N}_{2}$ Separation
}

\author{
Wan Zulaisa Amira Wan Jusoh, Sunarti Abdul Rahman* and Alia Aqilah Ghazali \\ ${ }^{1}$ Faculty of Chemical and Process Engineering Technology, \\ College of Engineering Technology, Universiti Malaysia Pahang, \\ Lebuhraya Tun Razak, 26300 Gambang, Kuantan, Pahang, Malaysia \\ "Corresponding author: sunarti@ump.edu.my
}

Published online: 25 November 2019

To cite this article: Wan Jusoh, W. Z. A., Abdul Rahman, S. \& Ghazali, A. A. (2019). Effect of adipic acid concentration on the isotactic polypropylene flat sheet using methyl salicylate prepared by TIPS for $\mathrm{CO}_{2} / \mathrm{N}_{2}$ separation. J. Phys. Sci., 30(3), 157-173, https://doi.org/10.21315/jps2019.30.3.10

To link to this article: https://doi.org/10.21315/jps2019.30.3.10

\begin{abstract}
Gas separation technology using membrane can effectively remove $\mathrm{CO}_{2}$ from flue gas as well as offer cost-effective installation. However, water vapour in flue gas causes the membrane to swell. Thus, this study focused on the enhancement of the durability and performance of membrane for $\mathrm{CO}_{2}$ and $\mathrm{N}_{2}$ separation where hydrophobic membranes were proposed with the introduction of adipic acid (AA) during preparation. The gas test was carried out using a pure gas permeation set up. A maximum $\mathrm{CO}_{2} / \mathrm{N}_{2}$ selectivity of 1.22 and $\mathrm{CO}_{2}$ permeance of 2388 GPU were obtained. Via scanning electron microscopy (SEM), a decreasing membrane pore size distribution was observed on isotactic polypropylene (iPP) with increasing AA in methyl salicylate (MS) membrane. Fourier transform infrared spectroscopy (FTIR) spectra showed no bonding changes between raw iPP and membrane fabricated via thermally induced phase separation (TIPS) method. The highest value of contact angle of the membranes prepared was $106^{\circ}$ without the addition of adipic acid. The hydrophobicity was observed to be escalating with the increase of nucleation agent.
\end{abstract}

Keywords: Thermally induced phase separation, isotactic polypropylene, methyl salicylate, hydrophobic membrane, $\mathrm{CO}_{2} / \mathrm{N}_{2}$ separation 


\section{INTRODUCTION}

Over the past few decades, the emission of flue gases into the atmosphere has increased and warmed the earth's surface. Excessive emission of flue gas into the environment is very dangerous to human health and harmful to other living organisms. Typically, the flue gas emission from natural gas, refinery gas or coal gas purification consists of $71.8 \%$ nitrogen $\left(\mathrm{N}_{2}\right), 13.6 \% \mathrm{CO}_{2}$ and $11.2 \%$ water vapour. ${ }^{1}$ One of the significant challenges in gas separation system is the presence of water vapour in flue gas. The presence of water vapour significantly decreases the permeability of gases. Although water vapour is usually considered a minor component, it can significantly affect the membrane's gas transport properties. ${ }^{2}$

Hydrophobic-based materials are best to prevent the intrusion of liquid into another side of the membrane. ${ }^{3,4}$ An ideal hydrophobic membrane should be highly hydrophobic and demonstrate high surface porosity, low resistant to mass transfer, and high resistant to chemicals at the feed streams. ${ }^{5}$ Improving the hydrophobicity of the membrane can increase the membrane long-term performance. ${ }^{6}$ Hydrophobicity provides a high specific surface area, enhances the selectivity performance, and increases the driving force of flux even at a low concentration at the feed. ${ }^{7}$ Hydrophobicity properties not only related to the chemical composition of a membrane surface, but also from its morphology or surface roughness. ${ }^{8}$

A recent study applied the mixed matrix membranes (MMMs) in the $\mathrm{CO}_{2} / \mathrm{N}_{2}$ separation with a very high separation factor. ${ }^{9}$ However, polymeric membrane fabrication is simpler and lower in cost compared with the MMMs. Isotactic polypropylene (iPP) particularly, is a low cost and highly hydrophobic membrane material with high mechanical strength, low molecular weight, and very thermally and chemically stable compared with other hydrophobic flat sheets. iPP also provides an excellent resistance to acids, alkalis and organic solvents. ${ }^{10}$ However, iPP cannot be dissolved in common solvent at low temperature due to its chemically inert property. iPP is a difficult material to be formed into membrane using typical solution casting method and requires for liquid-liquid demixing. ${ }^{11}$

There are two different methods available for liquid-liquid demixing either by changing temperature called thermally induced phase separation (TIPS) or by immersing the solution in a nonsolvent bath known as nonsolvent induced phase separation (NIPS). ${ }^{12}$ However, the NIPS method is less favourable due to the difficulty in controlling membrane porous structure compared with TIPS. ${ }^{13}$ The TIPS technique is known for its simplicity and easy to control the pores of membrane. In TIPS, to obtain a polymer-rich continuous matrix, the homogeneous polymer solution needs to undergo liquid-liquid demixing known as polymer-rich phase. This is the first step in the TIPS techniques. A dispersed polymer lean phase 
is an almost pure solvent. The next step in TIPS is the fixation step to fix the structure and mechanical stability of the membrane. This is needed to attain the crystallisation of the polymer-rich phase to become polymeric matrix. ${ }^{14}$

Prior to casting, the polymer is diluted to form a homogenous solution with suitable diluent. Typically, diluents used to dissolve the polypropylene have a very high melting point. Diphenyl ether (DPE), methyl salicylate (MS) and camphene are among the mostly used diluents. ${ }^{15,16}$ Due to the unpleasant odour of DPE and MS, there are other alteratives including a combination of soybean oil with diamyl phthalate (DAP). ${ }^{17}$ However, Tang et al. found that soybean oil had a weak compatibility with the iPP system. ${ }^{5}$ MS is a good diluent as it is low in molecular weight, miscible with polymer, less toxic, and demonstrates high thermal stability. ${ }^{18}$

Adipic acid (AA) is a well-known additive for matrix polymer as a nucleation agent. Nucleation agent acts as an accelerator for crystallisation rate and enhances the crystallisation temperature as well as generates more nuclei and spherulites pores structures. It is also important for the membrane to have a uniform microporous structure and thus increase the flux of the membrane. ${ }^{19}$ The preparation of membrane via TIPS typically forms narrow pores, which are also distributed to decrease membrane permeability.

The objective of this research work was to develop iPP membrane using MS diluent. The addition of AA is expected to improve the durability, morphological and $\mathrm{CO}_{2} /$ $\mathrm{N}_{2}$ gas separation performance. Single gas permeation was carried out to measure and evaluate the separation performance of gas pairs $\mathrm{CO}_{2} / \mathrm{N}_{2}$. In the current study, pure iPP and iPP/adipic acid were prepared and characterised using scanning electron microscopy (SEM), Fourier transform infrared (FTIR), and contact angle instrument to find the effect of AA on the morphological and separation properties of the membranes produced. In this study, AA was used and expected to enhance more nuclei and spherulites pores structures which constructed more uniform microporous structure and thus improved the hydrophobicity of the cheaper alternatives hydrophobic membrane, iPP. The durability was also expected to be enhanced.

\section{EXPERIMENTAL}

\subsection{Preparation of the Isotactic Polypropylene Membrane Using TIPS}

iPP with an average molecular weight of 250,000 was purchased from Sigma Aldrich, together with MS, AA and methanol. All the diluents purchased from 
Sigma Aldrich were used without further purification. Briefly, $20 \mathrm{wt} \%$ of iPP was mixed with $80-78.5 \mathrm{wt} \%$ of MS in beakers and $0.5-1.5 \mathrm{wt} \%$ of AA powder was also added. The beaker was placed on a hot plate at $180^{\circ} \mathrm{C}$ until a homogeneous solution formed. A little portion of the homogeneous solution was poured onto a casting plate as shown in Figure 1. The entire assembly was then quenched in a water bath to induce phase separation in the sample. Then, the membranes were immersed in methanol to extract the remaining diluents in the membranes. Microporous iPP membranes were obtained by evaporating the diluents in an oven at $65^{\circ} \mathrm{C}$ for $30 \mathrm{~min}$.

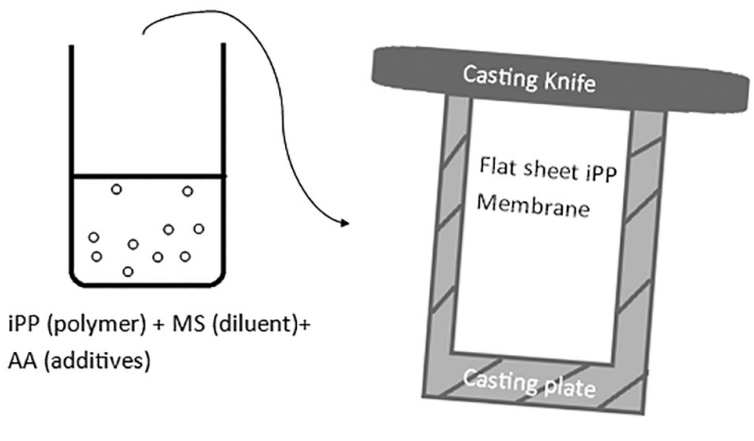

Figure 1: Preparation of the iPP membrane by TIPS method.

\subsection{Permeation of Carbon Dioxide and Nitrogen}

The performance of polypropylene membranes was tested with pure gases $\left(\mathrm{CO}_{2}\right.$ and $\mathrm{N}_{2}$ ) using a permeation test set. The thickness of the membrane prepared varied from $0.025 \mathrm{~cm}$ to $0.027 \mathrm{~cm}$. The membrane was cut into a small circle with a diameter of $4.8 \mathrm{~cm}$. Then, the circle membrane was pleated and folded around the permeate core. The size of the circle membrane must suite the permeate core to avoid any leakage of pure gas. The separation of pure gas across the fabricated membrane was tested at pressure range of 1-3 bar at the ambient temperature. The time taken for $20 \mathrm{~cm}^{3}$ of gas to permeate through the membrane was recorded four times.

The gas permeation using the sorption-diffusion theory is usually used where the productivity of a membrane is defined by the permeability of a gas through the membrane. The permeability of a gas, $i$, is given by Equation 1. Pure gas permeation tests were carried out as described elsewhere. ${ }^{20}$

$$
P_{i}=D_{i} \cdot S_{i}
$$


In Equation 1, $D_{i}$ and $S_{i}$ represent the diffusion and solubility coefficients of component $i$, respectively. Permeance can also be expressed as the flux normalised by film thickness $(l)$ and the transmembrane pressure $\left(\Delta P_{i}\right)$, as shown by Equation 2:

$$
P_{i}=(\text { Flux i } \times l) / \Delta P_{i}
$$

The unit for gas permeability is Barrer, where 1 Barrer $=\left(\mathrm{cm}^{3}(\mathrm{STP}) \cdot \mathrm{cm}\right) /$ $\left(\mathrm{cm}^{2} \cdot \mathrm{s} \cdot \mathrm{cm} \mathrm{Hg}\right) \times 10^{-10}$. When the thickness is difficult to define (often in the case with asymmetric membranes), the pressure normalised flux, or permeance $(P i / l)$ is used instead. The gas permeation unit is in GPU, where $1 \mathrm{GPU}=\left(\mathrm{cm}^{3}(\mathrm{STP})\right) /$ $\left(\mathrm{cm}^{2} . \mathrm{s} . \mathrm{cm} \mathrm{Hg}\right) \times 10^{-6}$. The separation selectivity $(\alpha)$ of a component is the ratio of the permeance obtained for the individual gases designated in Equation 3. For a mixture of gas $i$ and $j$, the ideal selectivity is described by:

$$
\alpha_{i j}=P_{i} / P_{j}
$$

where $P_{i}$ and $P_{j}$ are the permeabilities of components $i$ and $j$, respectively.

\subsection{Characterisation}

The cross section and surface layer were examined using SEM (Carl Zeiss EVO50). The other purpose of using this equipment is to examine the morphology or specifically the membrane structure, pore distribution, defects and presence of impurities. Chemical composition was tested using FTIR (Nicolet Avatar 370 DTGS). FTIR was performed to study the chemical structure of organic molecules and potential structural changes that occurred as a result of the membrane chemical treatment or degradation. Test System of JY-82 Video Contact Instrument was used to measure the contact angle of distilled water on the membrane. The contact angles were calculated from a digital video image of the drop on the membrane using an image processing program for the estimation of the contact angle from drop height and width. The contact angle on the membrane higher than $90^{\circ}$ indicates the hydrophobic membranes characteristic.

\section{RESULTS AND DISCUSSION}

\subsection{Permeability and Selectivity on the Fabricated Membranes}

Figure 2 shows the increase in permeate flux from 1700 GPU to 2300 GPU, $1380 \mathrm{GPU}$ to $1800 \mathrm{GPU}$, and $960 \mathrm{GPU}$ to $1100 \mathrm{GPU}$ for the applied pressures of 1.0 bar, 2.0 bar and 3.0 bar, respectively, when the additive was added up to $0.5 \mathrm{wt} \%$. The addition of adipic acid until $1.0 \mathrm{wt} \%$ slightly decreased the permeate 
flux. However, the permeate flux increased slightly when $1.5 \mathrm{wt} \%$ adipic acid was added until the permeances reached $1800 \mathrm{GPU}, 1370 \mathrm{GPU}$ and $940 \mathrm{GPU}$ for the applied pressures of $1.0 \mathrm{bar}, 2.0 \mathrm{bar}$ and $3.0 \mathrm{bar}$, respectively. The highest permeate flux was obtained from $0.5 \mathrm{wt} \%$ at 1 bar. The relative contributions of the different mechanism depend on the properties of the membranes and the gases, as well as the operating conditions like temperature and pressure. ${ }^{21}$ Theoretically, permeability is directly proportional to the pressure as shown in Equation 2. However, when the pressure was increased from 1 bar to 2 bar, the permeate flux gradually decreased. The highest permeability was obtained at 1 bar.

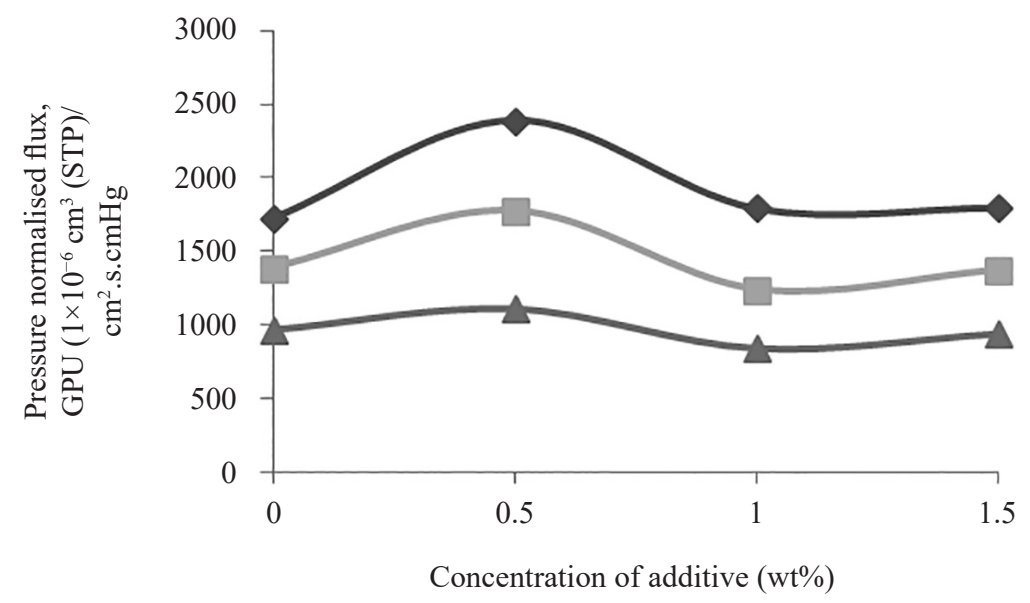

Figure 2: $\mathrm{CO}_{2}$ permeance for the membranes prepared using MS $(-$ denoting 1 bar, -2 bar, and -3 bar).

The permeance of $\mathrm{N}_{2}$ gas is shown in Figure 3. A similar result was observed as $0.5 \mathrm{wt} \%$ at 1 bar recorded the highest permeance for $\mathrm{N}_{2}$. The permeate fluxes of 1600 GPU, 1140 GPU and 900 GPU were recorded for the applied pressures of 1.0 bar, 2.0 bar and 3.0 bar, respectively, before additive was added. The permeate fluxes increased to $2240 \mathrm{GPU}, 1520 \mathrm{GPU}$ and $1190 \mathrm{GPU}$, respectively, when $0.5 \mathrm{wt} \%$ additive was added. When $1.5 \mathrm{wt} \%$ of additive was added, the respective permeate fluxes decreased until 1650 GPU, 1150 GPU and 850 GPU. Pressure can influence the permeance and also pore size of the membrane. ${ }^{22,23}$ However, the permeance of $\mathrm{N}_{2}$ and $\mathrm{CO}_{2}$ decreased when pressure was increased. The trends show the glassy properties of the fabricated iPP.${ }^{24}$ Glassy polymers membrane is expected to show a decline in permeance with pressure increase. ${ }^{25}$ 
The membrane prepared without adipic acid has smaller pore size compared to that with adipic acid. High permeance by smaller pore size membrane may be the results from the defects on the membrane surface. The adipic acid powder may not totally dissolved and incorporated inside the polymer matrix. Meanwhile, the permeance obtained by larger pore size membrane was due to the larger free volume. ${ }^{21}$ As the pore size decreases, the porosity also decreases, resulting in a lower gas flow through the membrane. Therefore, both average pore sizes and pore size distribution must be balanced to fabricate a good membrane and increase the flux of the membrane. ${ }^{26}$

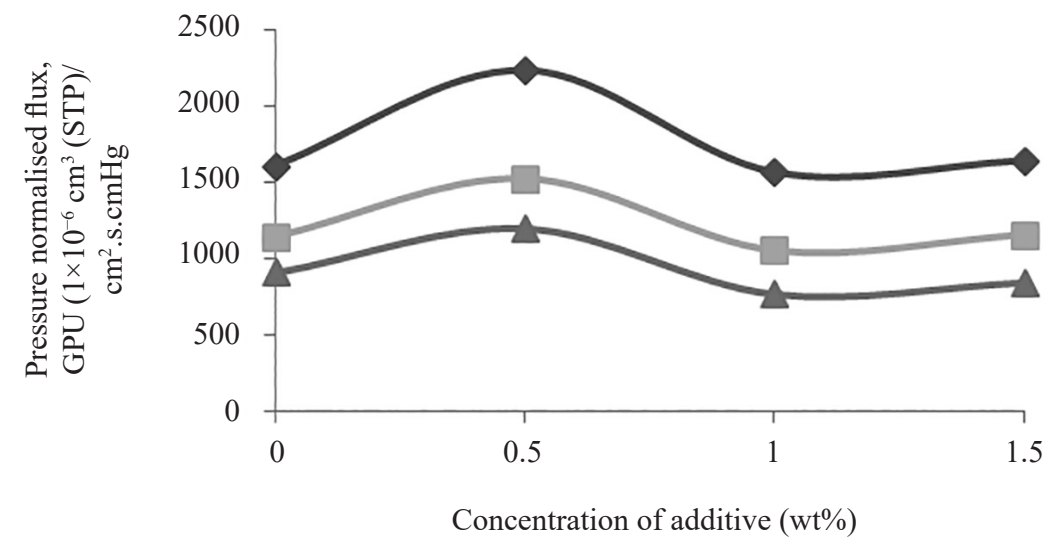

Figure 3: $\mathrm{N}_{2}$ permeance for the membranes prepared using MS ( - denoting 1 bar, 2 bar, and -3 bar).

Without the addition of a nucleation agent, the selectivities of 1.0, 1.22 and 1.10 were attained at the pressure of $1.0 \mathrm{bar}, 2.0 \mathrm{bar}$ and $3.0 \mathrm{bar}$, respectively (Figure 4). When the additive was increased to $0.5 \mathrm{wt} \%$, all the respective selectivities dropped to $1.1,1.2$ and 0.9. However, the respective selectivities gradually increased to $1.1,1.2$ and 1.09 when the additive was increased from $0.5 \mathrm{wt} \%$ to $1.5 \mathrm{wt} \%$. The best selectivity of 1.22 was obtained in the membrane without the additive at 1.5 bar. The results show that the selectivity of $\mathrm{N}_{2}$ was lower than that of $\mathrm{CO}_{2}$. Based on this, it can be concluded that $\mathrm{CO}_{2}$ has a smaller kinetic diameter than $\mathrm{N}_{2}$ which are $3.64 \AA$ and $3.3 \AA$, respectively. This makes the $\mathrm{CO}_{2}$ molecules to pass through the pores easier. As $\mathrm{CO}_{2}$ selectivity is found higher than $\mathrm{N}_{2}$, this condition is considered that gaseous permeate through membrane by molecular sieving mechanism. ${ }^{27}$ 


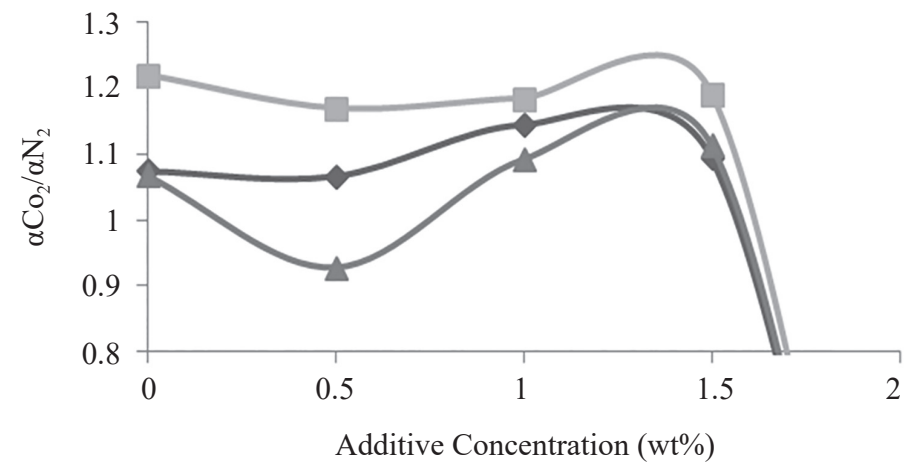

Figure 4: The relationship between additive concentration and selectivity of $\mathrm{CO}_{2}$ and $\mathrm{N}_{2}$ gas for the membranes prepared by MS ( $\checkmark$ denoting $1 \mathrm{bar},-2 \mathrm{bar}$, and 는.

A similar reported work on $\mathrm{CO}_{2} / \mathrm{N}_{2}$ separation performance revealed a $\mathrm{CO}_{2}$ permeance greater than 10 GPU.22 Based on the theory suggested by Robeson, permeability and selectivity possess a trade off an upper and lower boundary in Robeson plot. ${ }^{28}$ A plot of selectivity of $\mathrm{CO}_{2} / \mathrm{N}_{2}$ versus $\mathrm{CO}_{2}$ permeability of the fabricated membranes was constructed and compared to the Robeson plot (Figure 5). The highest $\mathrm{CO}_{2} / \mathrm{N}_{2}$ selectivity and $\mathrm{CO}_{2}$ permeability obtained by the membrane prepared using MS diluent were 1.7 Barrer and 180,000 Barrer, respectively. The low selectivity presented by the fabricated membranes indicates undesirable gas separation. ${ }^{29}$

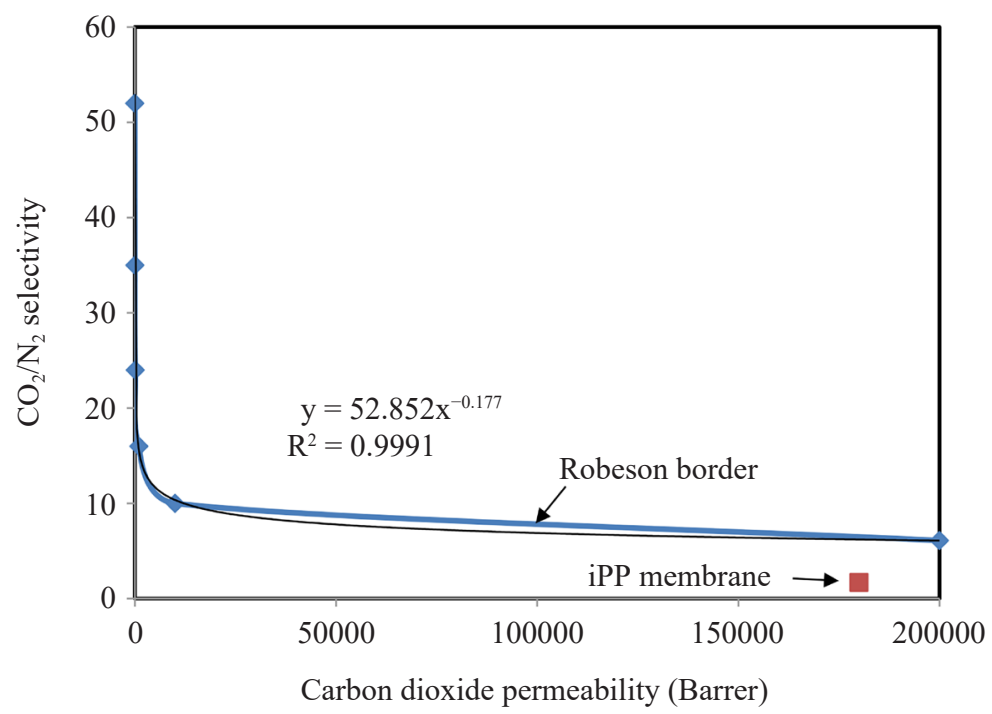

Figure 5: Selectivity of the fabricated membranes compared to the Robeson plot. 
The permeability of gases is higher in the iPP-MS membrane but it produces low selectivity. In gas separation, it is normal for membrane to have high permeability and low selectivity due to the viscous flow of the gas molecules through the iPPMS membrane, rather than diffusion. The pore size and pore size distribution affect the viscous flow and diffusion differently. ${ }^{30}$ Larger pores would allow the gases to pass through easily and faster compared to smaller pore size membranes, because of the viscous flow. Viscous flow would allow small molecules to pass through while diffuse flow occurs due to the diffusion of selective molecule gas through the membrane. The rate of gas molecules to pass through the iPP-MS membrane was considered high.

\subsection{Characterisation on the Structures and Morphology}

\subsubsection{Effect of adipic acid concentration on the membrane pore morphology}

Figure 6 presents the cross section of the membranes prepared using MS with the addition of $0.5 \mathrm{wt} \%$ and $1.5 \mathrm{wt} \%$ of adipic acid. The membranes obviously demonstrated pore sizes with thin walls and large diameter exemplifying the large interconnectivity between cellular pores. The polymer crystallisation kinetic such as pore size, porosity and pore size distribution can be controlled by adding suitable nucleating agent. ${ }^{31} \mathrm{~A}$ few researchers found that adipic acid was the preferable nucleating agent in the iPP membrane preparation. ${ }^{5,19}$ By adding the nucleating agent, more nuclei were enhanced thus spherulites pores structures and narrow pore sizes were accomplished. It is also believed that small pores contribute to higher hydrophobicity on the membrane surfaces. ${ }^{32}$

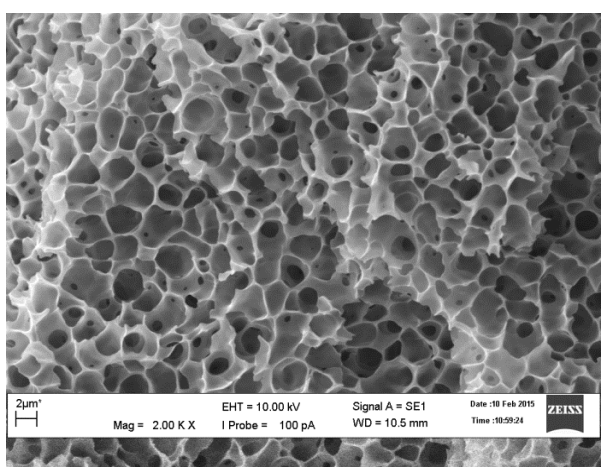

(a)

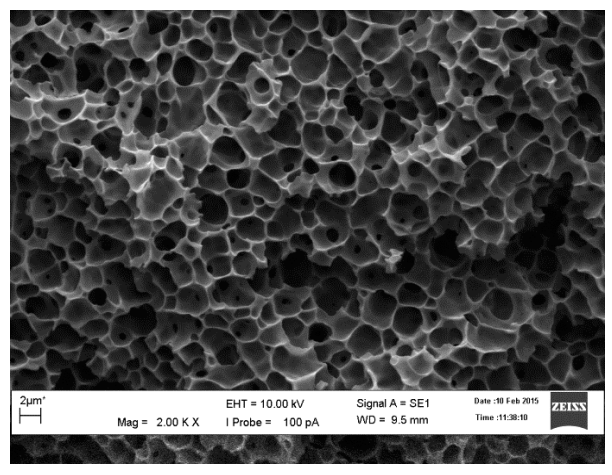

(b)

Figure 6: Membranes cross-sectional structures at 2000X magnification for (a) 0.5 , and (b) $1.5 \mathrm{wt} \%$ of AA. 
The membrane pores were loose at the beginning and then became compact with the increasing dosage of nucleating agent. The pore sizes increased with the increasing AA concentration. The internal pore sizes of the membranes prepared using MS diluent were estimated between 1.42-4.30 $\mu \mathrm{m}$ and 1.11-3.71 $\mu \mathrm{m}$ (Figure 7). The pore sizes in the membranes decreased with the increasing AA dosage. Luo et al. found development of smaller pore sizes membranes when the additive concentration was increased. ${ }^{31}$ According to Mansourizadeh and Ismail, a more uniform microporous structure would improve the hydrophobicity. ${ }^{7}$ Thus, it can be concluded that the addition of $1.5 \mathrm{wt} \%$ of adipic acid in the solution yielded a membrane with a uniform structure.
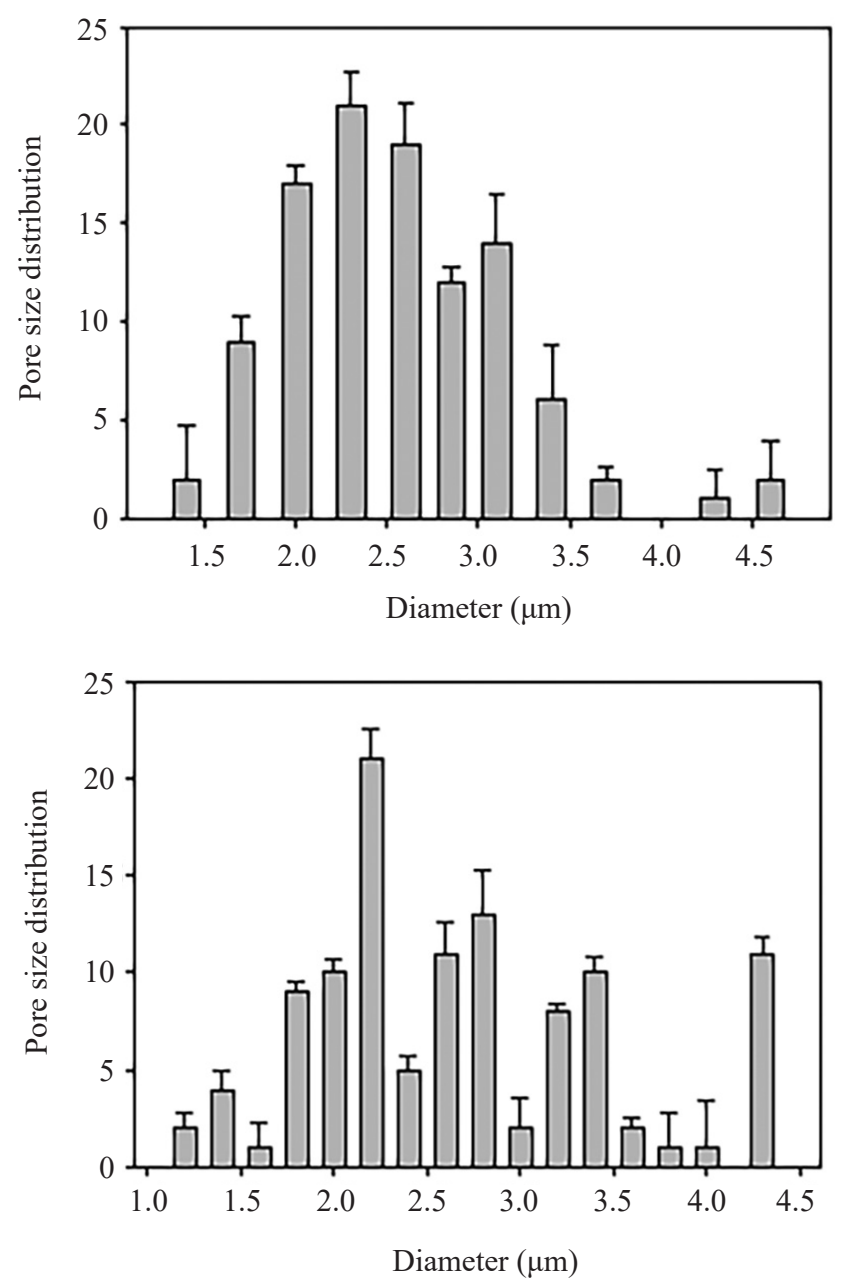

Figure 7: Pore size distribution of the iPP-MS membranes fabricated with (a) $0.5 \mathrm{wt} \%$, and (b) $1.5 \mathrm{wt} \%$ of adipic acid. 


\subsubsection{Chemical composition in membranes}

In Figure 8, two highest peaks were observed in the range of $2918-1920 \mathrm{~cm}^{-1}$ and $2952-2953 \mathrm{~cm}^{-1}$ indicating a strong intensity of $\mathrm{C}-\mathrm{H}$ bond. The spectrum ranges correspond to the $\mathrm{CH}_{3}, \mathrm{CH}_{2}$ and $\mathrm{CH}$ consisting of two or three bands. The third highest peaks are in the range of $1444-1457 \mathrm{~cm}^{-1}$ demonstrating the deformation of $\mathrm{CH}_{2}$ and $\mathrm{CH}_{3}$ with medium intensity. The peaks detected at $1376 \mathrm{~cm}^{-1}$ and $1375 \mathrm{~cm}^{-1}$ show a medium intensity of $\mathrm{CH}_{2}$ deformation. Variance and weak intensity peaks of $\mathrm{O}-\mathrm{H}$ bend from alcohol were detected at $756.50 \mathrm{~cm}^{-1}$ and $757.16 \mathrm{~cm}^{-1}$ for the membranes with $0.5 \mathrm{wt} \%$ and $1.0 \mathrm{wt} \%$ of additive, respectively. Two peaks at $755.23 \mathrm{~cm}^{-1}$ and $699.41 \mathrm{~cm}^{-1}$ in the membrane with $1.5 \mathrm{wt} \%$ of addictive indicate a weak alcohol group. The strong and medium bonds formed in the membranes show that the membranes produced were stable and high in strength which were expected to have high selectivity and permeability. So, the FTIR analysis supports the results in the permeation test.

From the FTIR analysis, it can be concluded that all the membranes contain peaks demonstrating a medium intensity of C-H bond. This alkane C-H stretching absorption just below $3000 \mathrm{~cm}^{-1}$ demonstrates the presence of saturated carbons.

\subsubsection{Contact angle}

Hydrophobic membrane can be achieved when the contact angle of the water droplet on the membrane is higher than $90^{\circ}$. When the contact angle is lower than $90^{\circ}$, the membrane is hydrophilic. ${ }^{33}$ The hydrophobicity of the produced membranes was measured at the top and bottom surfaces to determine which side is able to retain liquid better to be applied in gas separation applications. Figure 9 demonstrates the effect of additive concentration on the contact angle of the top and bottom side of the iPP-MS membrane. The highest contact angle of $106^{\circ}$ was obtained from the bottom side of the membranes prepared without the addition of adipic acid. Increasing the additive concentration from $0.5 \mathrm{wt} \%$ to $1.5 \mathrm{wt} \%$ increased the hydrophobicity of the membrane before it gradually decreased when $2.5 \mathrm{wt} \%$ of additive was added. This shows that increasing the nucleation agent will decrease the space for the pore growth and thus produces the narrow pore sizes membrane. ${ }^{19}$

The smaller pore diameter membranes are expected to resist the wetting problem better than the larger pore diameter membranes. The highest contact angle of $98^{\circ}$ was obtained from the membranes prepared using MS diluents. However, after adding more than $1.5 \mathrm{wt} \%$ of adipic acid, the water contact angle reduced gradually. This result may be due to drying process and surface roughness. ${ }^{34}$ The membranes prepared without additive gave the highest contact angle. However, some of the membranes had less than $90^{\circ}$ water contact angle. This was due to the solubility 

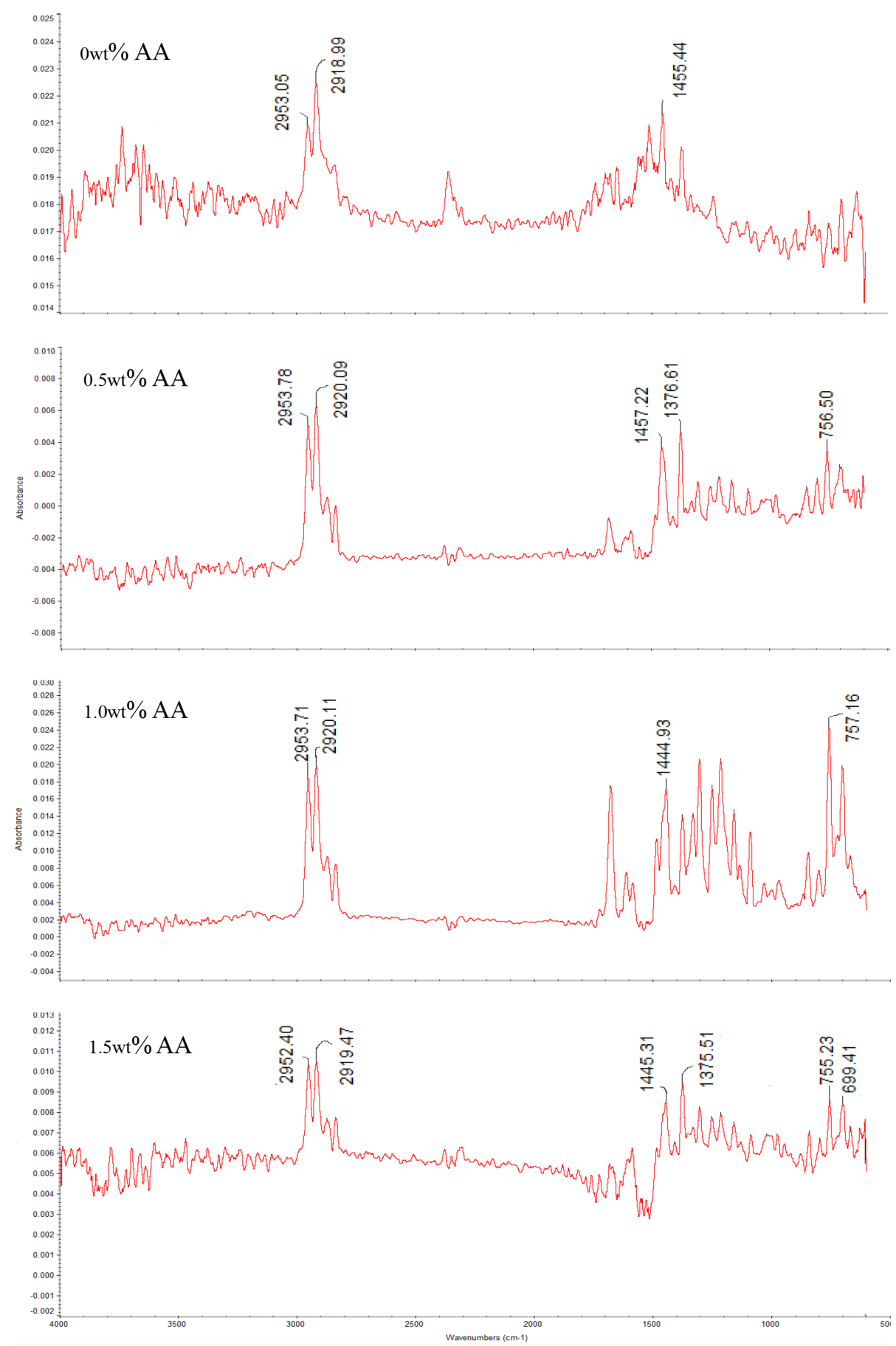

Figure 8: FTIR spectrum of iPP-MS membranes. 
differences of iPP-MS system diluent, which was larger and less compatible. The preparation of membrane using MS caused the polymer solution to have a lower viscosity that contributed to the larger particle sizes in membrane due to slower pore growth rate. ${ }^{15}$ It is obvious that the bottom side of the membranes was better than the top side of the membrane. It may be associated with the smaller pore size at the bottom side. Small pore size is attributed by a very fast cooling rate. ${ }^{35}$ The membranes prepared without additive were able to produce a very high contact angle compared to the membranes with additives. There was no significant effect on hydrophobicity discovered by adding $1.5 \mathrm{wt} \%$ of adipic acid compared to the membrane prepared without adipic acid, which also shows that adipic acid contributed less effect on the nucleation. It can be concluded that adipic acid was not a good nucleating agent for the iPP-MS system.

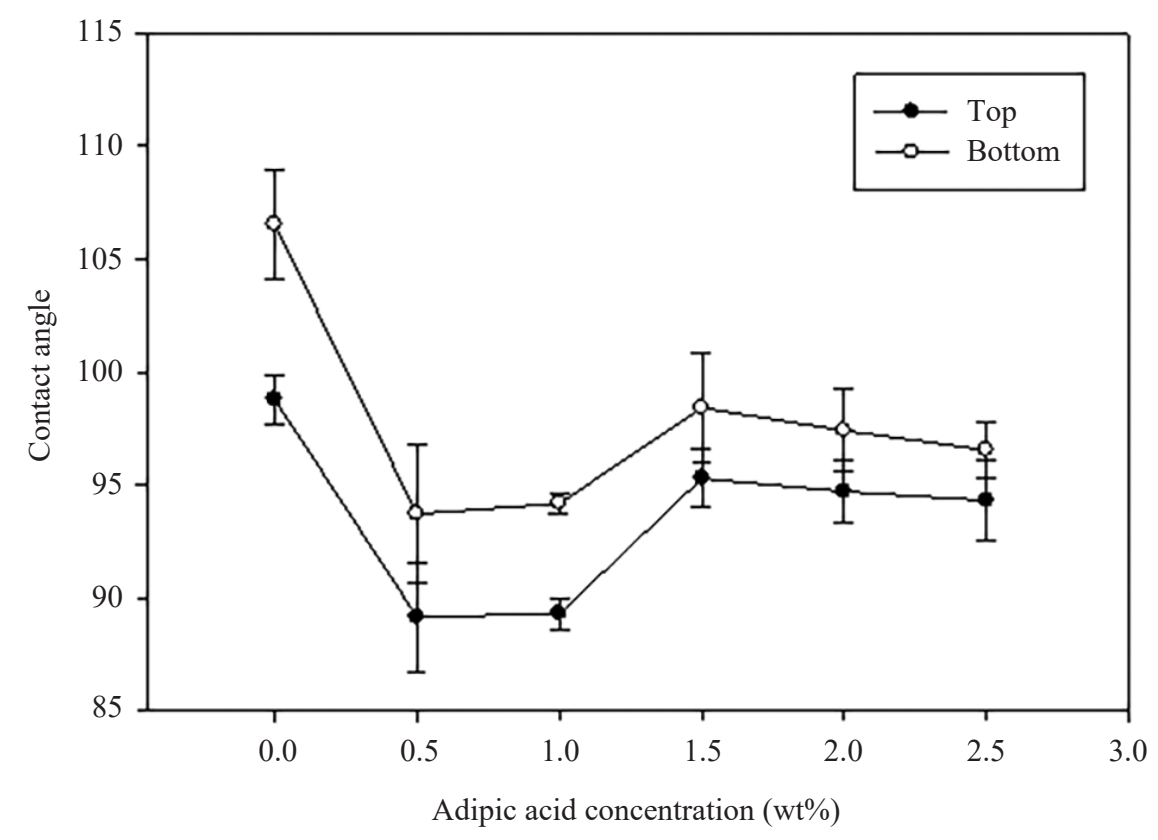

Figure 9: The effect of additive concentration on the contact angle measurement of the membrane surfaces.

The high hydrophobicity is mainly associated by smaller pore size, meanwhile, high permeability is attributed by the large pore size. However, adding adipic acid would increase the pore growth and eventually narrow down the pore size. Moreover, hydrophobicity does not only depend on pore size but also other factors such as surface roughness. 


\section{CONCLUSION}

Adding adipic acid into polymer solution developed a high permeate flux of $\mathrm{CO}_{2}$ and $\mathrm{N}_{2}$ gases. The highest membrane porosity and flux performance were achieved by the membrane with $0.5 \mathrm{wt} \%$ of adipic acid tested at $1 \mathrm{bar}$. The permeabilities for $\mathrm{CO}_{2}$ and $\mathrm{N}_{2}$ obtained were $2300 \mathrm{GPU}$ and $2240 \mathrm{GPU}$, respectively. The highest selectivity of 1.22 was demonstrated by the membrane without additive. $\mathrm{CO}_{2}$ selectivity was larger than $\mathrm{N}_{2}$ selectivity. The FTIR analysis shows that all the membranes had peaks of strong and medium intensity of the $\mathrm{C}-\mathrm{H}$ bonds. There was a variance and weak intensity of $\mathrm{O}-\mathrm{H}$ bend from alcohol detected in the membranes with additive may be due to the smaller pores by formed in the membrane which required some time for solvents and diluents to totally evaporate from the pores. The highest contact angle obtained was $106^{\circ}$ in the membranes without additive. However, the high hydrophobicity value does not guarantee a high gas separation due to other possible factors.

\section{ACKNOWLEDGEMENTS}

The authors wish to thank Universiti Malaysia Pahang, Malaysia for the financial support for the publication of this research article (grant PGRS180323).

\section{REFERENCES}

1. $\mathrm{Xu}, \mathrm{X}$. et al. (2003). Separation of $\mathrm{CO} 2$ from power plant flue gas using a novel $\mathrm{CO}_{2}$ "molecular basket" adsorbent. ACS Div. Fuel Chem., 48(1), 162-163, https://doi.org/10.1016/j.jrp.2007.09.007.

2. Chen, G. Q. et al. (2011). Water vapor permeation in polyimide membranes. J. Membr. Sci., 379(1-2), 479-487, https://doi.org/10.1016/j.memsci.2011.06.023.

3. Yan, S. P. et al. (2007). Experimental study on the separation of $\mathrm{CO}_{2}$ from flue gas using hollow fiber membrane contactors without wetting. Fuel Process. Technol., 88(5), 501-511, https://doi.org/10.1016/j.fuproc.2006.12.007.

4. Zhang, H. Y. et al. (2008). Theoretical and experimental studies of membrane wetting in the membrane gas-liquid contacting process for $\mathrm{CO}_{2}$ absorption. J. Membr. Sci., 308(1-2), 162-170, https://doi.org/10.1016/j.memsci.2007.09.050.

5. Tang, N. et al. (2010). Preparation and morphological characterization of narrow pore size distributed polypropylene hydrophobic membranes for vacuum membrane distillation via thermally induced phase separation. Desalin., 256(1-3), 27-36, https://doi.org/10.1016/j.desal.2010.02.024.

6. Abdulmunem, M. et al. (2017). Carbon dioxide capture using a superhydrophobic ceramic hollow fibre membrane for gas-liquid contacting process. J. Clean. Prod., 140, 1731-1738, https://doi.org/10.1016/j.jclepro.2016.07.015. 
7. Mansourizadeh, A. \& Ismail, A. F. (2010). Effect of $\mathrm{LiCl}$ concentration in the polymer dope on the structure and performance of hydrophobic PVDF hollow fiber membranes for $\mathrm{CO}_{2}$ absorption. Chem. Eng. J., 165(3), 980-988, https://doi.org/10.1016/j.cej.2010.10.034.

8. Franco, J. A. et al. (2008). Fabrication of a superhydrophobic polypropylene membrane by deposition of a porous crystalline polypropylene coating. J. Membr. Sci., 318(1-2), 107-113, https://doi.org/10.1016/j.memsci.2008.02.032.

9. Fernández-barquín, A., Casado-coterillo, C. \& Irabien, Á. (2017). Separation of $\mathrm{CO}_{2}-\mathrm{N}_{2}$ gas mixtures: Membrane combination and temperature influence. Sep. Purif. Technol., 188, 197-205, https://doi.org/10.1016/j.seppur.2017.07.029.

10. Yave, W. \& Quijada, R. (2008). Preparation and characterization of porous microfiltration membranes by using tailor-made propylene/1-octadecene copolymers. Desalin., 228(1-3), 150-158, https://doi.org/10.1016/j. desal.2007.09.006.

11. Pabby, A. K. \& Sastre, A. M. (2013). State-of-the-art review on hollow fibre contactor technology and membrane-based extraction processes. J. Membr. Sci., 430, 263-303, https://doi.org/10.1016/j.memsci.2012.11.060.

12. Jung, J. T. et al. (2016). Understanding the non-solvent induced phase separation (NIPS) effect during the fabrication of microporous PVDF membranes via thermally induced phase separation (TIPS). J. Membr. Sci., 514, 250-263, https://doi.org/10.1016/j.memsci.2016.04.069.

13. Vanegas, M. E., Quijada, R. \& Serafini, D. (2009). Microporous membranes prepared via thermally induced phase separation from metallocenic syndiotactic polypropylenes. Polym., 50(9), 2081-2086, https://doi.org/10.1016/j. polymer.2009.03.006.

14. Shokri, E. \& Yegani, R. (2012). Full-factorial experimental design to determine the impacts of influential parameters on the porosity and mechanical strength of LLDEP microporous membrane fabricated via thermally induced phase separation method. J. Membr. Sep. Technol., 1, 43-51, https://doi.org/10.6000/19296037.2012.01.01.6.

15. Matsuyama, H. et al. (2000). Formation of polypropylene particles via thermally induced phase separation. Polym., 41(24), 8673-8679, https://doi.org/10.1016/ S0032-3861(00)00268-8.

16. Zulaisa, W. A. N., Wan, A. \& Rahman, S. A. (2015). Development of hydrophobic microporous isotactic polypropylene membrane for membrane contactor application. Adv. Mater. Res., 1113, 36-42, https://doi.org/10.4028/www.scientific. net/AMR.1113.36.

17. Luo, B. et al. (2008). Formation of anisotropic microporous isotactic polypropylene (iPP) membrane via thermally induced phase separation. Desalin., 233(1-3), 19-31, https://doi.org/10.1016/j.desal.2007.09.023.

18. Cui, Z. et al. (2013). Poly(vinylidene fluoride) membrane preparation with an environmental diluent via thermally induced phase separation. J. Membr. Sci., 444, 223-236, https://doi.org/10.1016/j.memsci.2013.05.031. 
19. Kim, I. C., Yun, H. G. \& Lee, K. H. (2002). Preparation of asymmetric polyacrylonitrile membrane with small pore size by phase inversion and posttreatment process. J. Membr. Sci., 199(1), 75-84, https://doi.org/10.1016/S03767388(01)00680-9.

20. Khadijah, S. \& Harun, Z. (2016). Chemical engineering research and design preparation and characterization of low cost porous ceramic membrane support from kaolin using phase inversion/sintering technique for gas separation: Effect of kaolin content and non-solvent coagulant bath. Chem. Eng. Res. Des., 112, 24-35, https://doi.org/10.1016/j.cherd.2016.06.007.

21. Pandey, P. \& Chauhan, R. S. (2001). Membranes for gas separation. Progr. Polym. Sci., 26(6), 853-893, https://doi.org/10.1016/S0079-6700(01)00009-0.

22. Huang, Y., Merkel, T. C. \& Baker, R. W. (2014). Pressure ratio and its impact on membrane gas separation processes. J. Membr. Sci., 463, 33-40, https://doi. org/10.1016/j.memsci.2014.03.016.

23. Shi, T. et al. (2018). Effect of pore size on the carbon dioxide adsorption behavior of porous liquids based on hollow silica. Chem. Phys. Chem., 19, 130-137, https:// doi.org/10.1002/cphc. 201700842 .

24. Mileva, D. et al. (2012). Homogeneous nucleation and mesophase formation in glassy isotactic polypropylene. Polym., 53(2), 277-282, https://doi.org/10.1016/j. polymer.2011.11.064.

25. Matteucci, S. et al. (2006). Transport of gases and vapors in glassy and rubbery polymers. In Yampolskii, Y. et al. (eds.), Materials science of membranes for gas and vapor separation. New York: Wiley, 1-47, https://doi.org/10.1002/047002903X. ch1.

26. Dorosti, F., Omidkhah, M. \& Abedini, R. (2015). Enhanced $\mathrm{CO}_{2} / \mathrm{CH}_{4}$ separation properties of asymmetric mixed matrix membrane by incorporating nano-porous ZSM-5 and MIL-53 particles into Matrimid®5218. J. Nat. Gas Sci. Eng., 25, 88-102, https://doi.org/10.1016/j.jngse.2015.04.033.

27. Yang, H. et al. (2008). Progress in carbon dioxide seperation and capture: A review. J. Environ. Sci., 20, 14-27, https://doi.org/10.1016/S1001-0742(08)60002-9.

28. Robeson, L. M. (2008). The upper bound revisited. J. Membr. Sci., 320, 390-400, https://doi.org/10.1016/j.memsci.2008.04.030

29. Scholes, C. A., Kentish, S. E. \& Stevens, G. W. (2008). Carbon dioxide separation through polymeric membrane systems for flue gas applications. Rec. Pat. Chem. Eng., 1, 52-66, https://doi.org/10.2174/1874478810801010052.

30. Geistlinger, H. et al. (2015). The impact of pore structure and surface roughness on capillary trapping for 2-D and 3-D porous media: Comparison with percolation theory. Wat. Res. Res., 51, 4923-4947, https://doi.org/10.1002/2015WR017173.

31. Luo, B. et al. (2006). Effects of nucleating agents and extractants on the structure of polypropylene microporous membranes via thermally induced phase separation. Desalin., 192(1-3), 142-150, https://doi.org/10.1016/j.desal.2005.10.013.

32. Lv, Y. et al. (2012). Fabrication and characterization of superhydrophobic polypropylene hollow fiber membranes for carbon dioxide absorption. Appl. Energy, 90(1), 167-174, https://doi.org/10.1016/j.apenergy.2010.12.038. 
33. Dindore, V. Y. et al. (2004). Membrane-solvent selection for $\mathrm{CO}_{2}$ removal using membrane gas-liquid contactors. Sep. Purif. Technol., 40(2), 133-145, https://doi. org/10.1016/j.seppur.2004.01.014.

34. Yave, W. et al. (2005). Syndiotactic polypropylene as potential material for the preparation of porous membranes via thermally induced phase separation (TIPS) process. Polym., 46(25), 11582-11590, https://doi.org/10.1016/j. polymer.2005.10.012.

35. Wang, B. et al. (2018). Porous membranes prepared by a combined crystallisation and diffusion (CCD) method: Study on formation mechanisms. J. Membr. Sci., 548, 136-148, https://doi.org/10.1016/j.memsci.2017.11.005. 